\title{
EMERGENCE AND ANTIBIOTIC SENSITIVITY PATTERN OF ACINETOBACTER BAUMANNII IN HOSPITAL FACILITY
}

\author{
DINOBANDHU NANDI, ANANIA ARJUNA* \\ Department of Medical Laboratory Sciences, Lovely Professional University, Phagwara, Punjab, India. Email: anania.arjuna@lpu.co.in
}

Received: 18 October 2016, Revised and Accepted: 30 November 2016

\begin{abstract}
Objective: Nosocomial infections or Hospital acquired infection (HAI) are one of the major threats to hospitalized patients as well as for the hospital associated personnel. In last few years there is a gross change in causative agents, new organisms have come out with great threat to hospitals as they possess antibiotic resistance property e.g. production of biofilm, production of enzymes such as $\beta$ - lactamases. Among many organisms, Acinetobacter baumannii has emerged as a potent nosocomial pathogen. Our objective of this study was to find the burden of Acinetobacter baumannii infections which are associated as nosocomial infections and to determine the drug of choice for an effective treatment.
\end{abstract}

Methods: Clinical specimens were collected from patients of different unit of the hospital by maintaining universal precautions and standard microbiological protocols. All the respective specimens were cultured in respective culture medium i.e. MacConkey agar, blood agar, chocolate agar, cysteine lactose electrolyte deficient (CLED) agar and, fluid thioglycolate (TG) medium at $37^{\circ} \mathrm{C}$ for $24-48$ hours. After incubation of $24-48$ hours culture plates were examined for bacterial growth and identification and antibiotic sensitivity test was made by Vitek2 compact.

Result: The study was conducted at the department of microbiology from January 2016 to April 2016 . A total of 2582 specimens were collected and processed for identification and sensitivity testing. Specimens of all age group ( 2 days- 93 years) and both sexes were processed for identification of A. baumannii and antibiotic sensitivity testing. A total of 119 isolates (4.60\%) of A. baumannii were obtained from 2582 clinical specimens. The most common infection A. baumannii was found as lower respiratory tract infection (89.07\%) followed by abscess (6.72\%), septicaemia (2.52\%), urinary tract infections $(0.84 \%)$, and soft tissue infections $(0.84 \%)$. The maximum sensitivity of $A$. baumannii isolates were seen to Colistin (CL) (119, $100 \%)$, followed by Tigecycline (TGC) $(63,52.94 \%)$ and Minocycline (MIN) (27, 22.69\%). The maximum resistant was observed for Imipenem (IMI), Aztreonam (AZT) and Ticarcillin- clavulanic acid (TIC) (119, 100\%).

Conclusion: The Gram- negative coccobacillus, Acinetobacter baumannii poses a formidable threat to patients. It has emerged as a superbug in hospital environment particularly in ICU units. The chances of A. baumannii infections increase in the presence of iatrogenic factors like inadequate long- term antibiotic therapy and new interventions in a medical facility. To control the burden of Acinetobacter infections new therapies such as combine therapy must be obtained and followed with proper dose as recommend by physicians; along with awareness of the importance of this infection should be implicated. Proper sanitation, good housekeeping, sterilization of equipment, hand hygiene, water purification, isolation procedures and maintaining of the hospital environment, use of infection control practices are some of the measures to control the transmission of Acinetobacter spp. among hospital personnel.

Keywords: Acinetobacter baumannii, Biofilm, $\beta$-lactamases, Hospital acquired infection.

(C) 2017 The Authors. Published by Innovare Academic Sciences Pvt Ltd. This is an open access article under the CC BY license (http://creativecommons. org/licenses/by/4. 0/) DOI: http://dx.doi.org/10.22159/ajpcr.2017.v10i3.15742

\section{INTRODUCTION}

Hospital acquired infection (HAI) also called nosocomial infection is a kind of infection which is acquired in the hospital by a patient in whom the infection was not present at the time of admission but emerged after discharging and it also includes occupational infections among the staff of the organization [1,2]. Nosocomial infections are mostly caused by Gram-negative organisms and are one of the major issues in patient safety. HAIs are one of the major causes of death [3] which have worldwide prevalence and affect developed, developing and resourcepoor countries. The rate of morbidity and mortality is significantly increased and cause financial losses for health-care system. The study has revealed that within hospital it is mostly prevalent is intensive care units (ICU) followed by acute surgical and orthopedic wards [4], approximately $30 \%$ patients in ICU are affected by at least one nosocomial infection [5]. Acinetobacter is Gram-negative coccobacillus, who has become the most prevalent cause of HAI. It has been identified to cause sporadic infections in health-care settings and has a significant role in colonization in critically ill patients [6]. The species Acinetobacter are associated with healthcare-related outbreaks [7-9]. Advance medical interventions and various surgical procedures increase the rate of nosocomial infections. Among all species of Acinetobacter, Acinetobacter baumannii has been found to cause approximately
$80 \%$ of reported Acinetobacter infections [10]. Their rapid ability to acquire resistant genes, minimal nutritional requirement, adherence to inanimate objects and long-term survival on them, biofilm production made them a successful nosocomial pathogen that is associated with respiratory tract infections, abscesses, bloodstream infections, meningitis, urinary tract infections, pleural effusion, etc. They resist mostly all common antibiotics through a number of different mechanisms which includes production of enzymes ( $\beta$-lactamases, aminoglycosides-modifying enzymes), alteration in outer membrane proteins and penicillin-binding proteins, efflux pumps, e.g., multidrug efflux pump, i.e., AdeABC, MATE pump AdeM, Tet (A), and Tet (B). Lower respiratory tract infections were more prevalent $(89.07 \%)$ in hospital facility followed by abscess (6.72\%), septicemia (2.52\%), urinary tract infections $(0.84 \%)$, and soft tissue infections $(0.84 \%)$. Males were most commonly affected with Acinetobacter infections (64.70\%) when compared with females (35.30\%). A more frequent visit to the hospitals might be the possible cause of infections for the males [11]. Colistin was the most promising drug followed by tigecycline and minocycline. Imipenem, aztreonam, and ticarcillin-clavulanic acid were most resistant. Good housekeeping, sterilization of equipment, hand hygiene, water purification, isolation procedures and maintaining of the hospital environment, use of infection control practices should be implemented to control the rate $A$. baumannii infection. 
Table 1: Gender wise distribution of $A$. baumannii infections

\begin{tabular}{lll}
\hline Gender & Total cases & Positive cases \\
\cline { 3 - 3 } & & $\mathbf{n}(\%)$ \\
\hline Male & 1718 & $77(64.70)$ \\
Female & 864 & $42(35.30)$ \\
Total & 2582 & $119(100)$ \\
\hline
\end{tabular}

A. baumannii: Acinetobacter baumannii
The study was conducted at the department of microbiology from January 2016 to April 2016. A total of 2582 clinical specimens were collected from patients of different unit of the hospital by maintaining universal precautions and standard microbiological protocols. The specimens include lower respiratory tract samples, i.e., endotracheal secretions, tracheal secretions, bronchoalveolar lavage, sputum, bronchial wash, and endotracheal tract tips, pus and pus swabs, blood, cerebrospinal fluid, pleural fluid, ascitic fluid, and tissues. All specimens were cultured on MacConkey agar and sheep blood agar (except

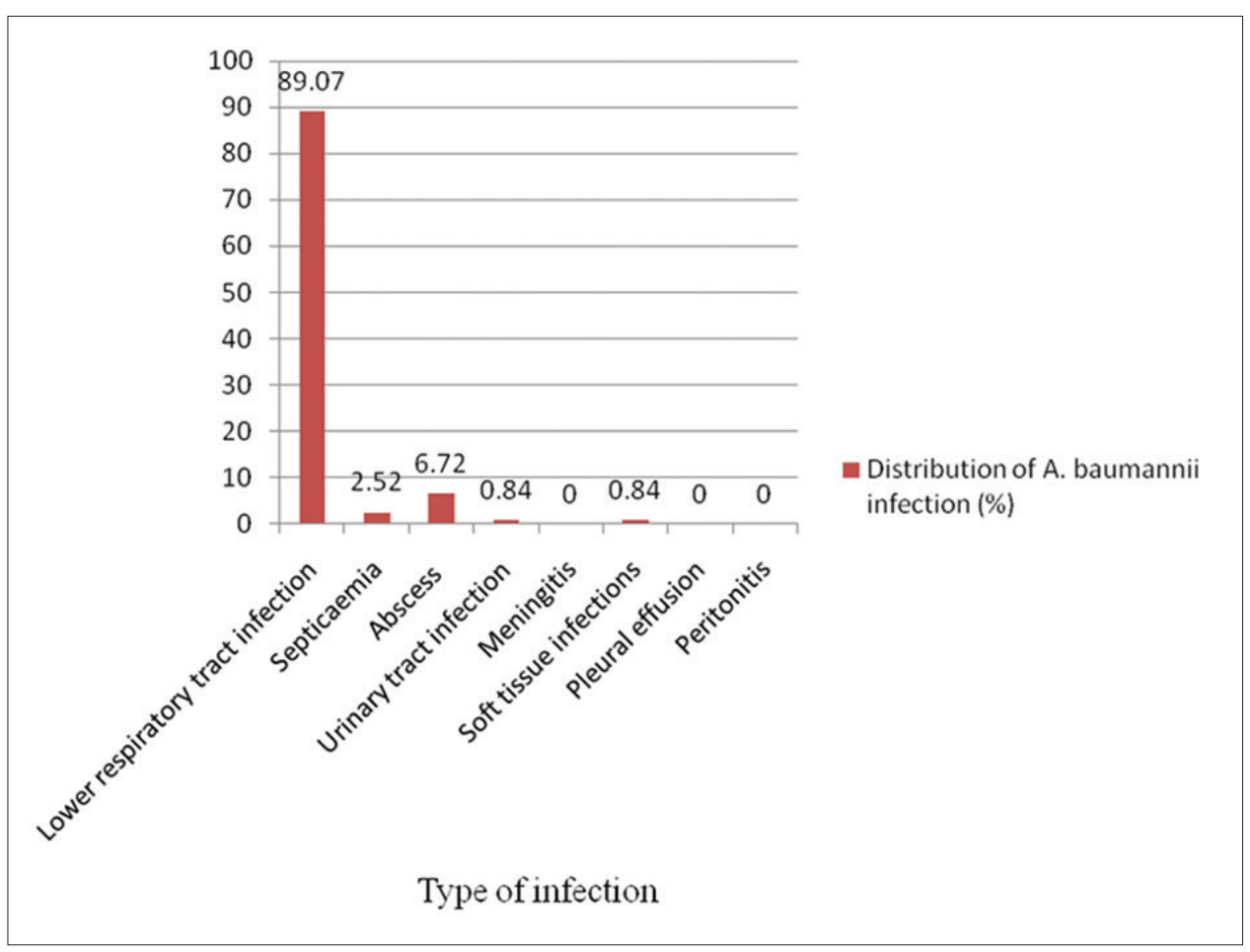

Fig. 1: Distribution of Acinetobacter baumannii infections

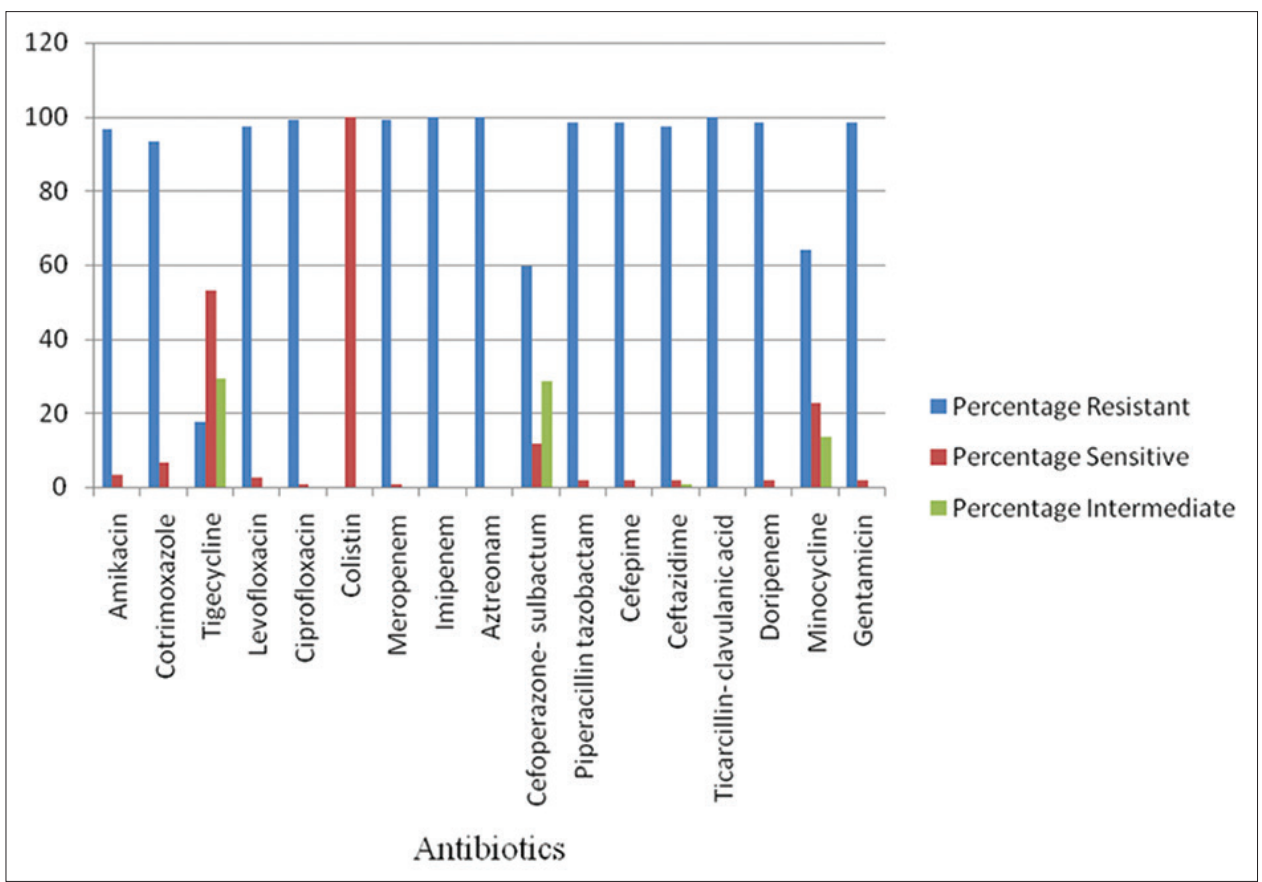

Fig. 2: Effect of antibiotics on Acinetobacter baumannii isolates 
urine, it was inoculated on cystine lactose electrolyte deficient agar) and incubated at $37^{\circ} \mathrm{C}$ for $24-48$ hrs. On MacConkey agar nonlactose fermenter colonies were obtained with faint pink color due to oxidation of lactose by the organism. Translucent to opaque convex colonies were obtained on sheep blood agar. On cystine lactose electrolyte deficient agar colonies were blue in color. The colonies were further tested for oxidase which was negative. The identification and sensitivity were done by Vitek2 compact which is based on advanced colorimetry and MIC technique, respectively.

A total of 2582 specimens were collected and processed for identification and sensitivity testing. Specimens of all age group (2 days-93 years) and both sexes were processed for identification of $A$. baumannii and antibiotic sensitivity testing. A total of 119 isolates $(4.60 \%)$ of A. baumannii were obtained from 2582 clinical specimens. The most common infection $A$. baumannii was found as lower respiratory tract infection $(89.07 \%)$ followed by abscess $(6.72 \%)$, septicemia $(2.52 \%)$, urinary tract infections $(0.84 \%)$, and soft tissue infections $(0.84 \%)$ (Fig. 1).

Out of 2582 specimens processed 1718 (66.54\%) were from male with positive A. baumannii infections 77 (64.70\%) and 864 (33.46\%) specimens were from female with $42(35.30 \%)$ positive A. baumannii infections. Males (64.70\%) were more commonly infected with A. baumannii than females (35.30\%) (Table 1).

The maximum sensitivity of $A$. baumannii isolates were seen to colistin $(119,100 \%)$, followed by tigecycline $(63,52.94 \%)$ and minocycline $(27,22.69 \%)$ whereas the sensitivity to cefoperazone-sulbactam was $11.8 \%$. Isolates were appeared to be intermediately sensitive to tigecycline $(35,29.41 \%)$, cefoperazone-sulbactam $(34,28.6 \%)$, minocycline $(16,13.45 \%)$, and ceftazidime $(1,0.84 \%)$. The maximum resistant was observed for imipenem, aztreonam, and ticarcillinclavulanic acid $(119,100 \%)$, followed by ciprofloxacin $(118,99.16 \%)$, meropenem (118, 99.16\%), piperacillin-tazobactam (117, 98.32\%), cefepime (117, 98.32\%), doripenem (117, 98.32\%), gentamicin $(117,98.32 \%)$, and levofloxacin $(117,97.48 \%)$ (Fig. 2).

A. baumannii is Gram-negative coccobacillus that has emerged as a successful nosocomial pathogen in past two decades. Its ability to resist drug, minimal nutrition requirements and ubiquitous distribution in nature made it a superbug. They are ubiquitous inhabitants of soil, water, and sewage environments. It is a commensal flora of human and animal skin that eases its transmission to critically ill patients in a hospital facility. Advance facilities in treatment and use of broad-spectrum antibiotic therapy increases the rate of morbidity and mortality of Acinetobacter infections. Acinetobacter adheres to surfaces and survives for a long time on surfaces of inanimate substances which play a key role in their pathogenesis. They are a strong producer of biofilm which helps them in trapping and concentrating all essential requirements from the environment and also provide a gate for horizontal gene transfer. Their ability of rapid acquiring genes that encoded drug resistant property has made them a potent pathogen in a hospital environment. Biofilm prevents bacteria from host immune defense and also diminish the action of antibiotics. The ratio of multi-drug resistant $A$. baumannii is increased, particularly in ICU patients [12]. In last two decades, the incidence of Acinetobacter infections has increased at a high rate that made them be ranked as $2^{\text {nd }}$ most common nosocomial pathogen found in all clinical specimens after Pseudomonas aeruginosa and $4^{\text {th }}$ according to the frequency of infections after P. aeruginosa, Staphylococcus aureus, and Klebsiella pneumoniae [13].

In this study, a total of 119 (4.60\%) A. baumannii isolates were isolated using Vitek2 compact from a total of 2582 processed clinical specimens including lower respiratory samples, blood, urine, body fluids, and tissues. Respiratory tract infections (89.07\%) were most common infection observed followed by abscess $(6.72 \%)$, septicemia $(2.52 \%)$, urinary tract infections $(0.84 \%)$, and soft tissue infections $(0.84 \%)$ (Figure 1). The reason for the respiratory infections to be more frequent is may be because of the use of mechanical ventilators and intubations. Tripathi et al. reported a total number of 107 (1.02\%) Acinetobacter isolates from all processed clinical specimens and Mal-Warid and Thahab reported $11(2.40 \%)$ isolates of $A$. baumannii from a total of 458 clinical samples which is well comparable with our study.

In this study, A. baumannii infections were common in males $(64.70 \%)$ as compared with females $(35.30 \%)$. A more frequent visit to the hospitals might be the possible cause of infections for the males [11].

The notorious nature of $A$. baumannii to acquire resistant genes is accounting for drug resistance to most common antibiotics. This has created a major challenge in patient safety. Acinetobacter spp. is universally resistant to penicillin, ampicillin, and cephalothin. [11]. Acinetobacter resists mostly all the classes of drug including $\beta$-lactams, aminoglycosides, quinolones, and tetracyclines. Through the production of enzymes ( $\beta$-lactamases, aminoglycosides-modifying enzymes), changes in their OMPs, using of multidrug efflux pumps and alteration in the affinity or expression of PBPs Acinetobacter spp. resist $\beta$-lactams and carbapenems. In this study, IMI, azidothymidine, and TIC have shown maximum resistant (100\%) followed by CIP (99.16\%), MER (99.16\%), PT (98.32\%), CPM (98.32\%), DOR (98.32\%), GEN (98.32\%), and LEV (97.48\%). Colistin was the most sensitive drug (100\%) obtained. Sensitivity to tight glycemic control (TGC) and MIN was $52.94 \%$ and $22.69 \%$, respectively. Isolates were appeared to be intermediately sensitive to TGC (29.41\%), CFS (28.6\%), MIN (13.45\%), and CAZ (0.84\%). Development of new therapies, well-managed clinical trials of existing antibiotics for combination therapy, prevention of transmission of the hospital-associated infections, hospital hygiene are essential to control Acinetobacter infections.

\section{REFERENCES}

1. Ducel G, Fabry J, Nicolle L. Guide Pratique Pour la Lute Contre l' Infection Hospitalière. WHO/BAC/79.1. Geneva: WHO; 2002.

2. Benenson AS. Control of Communicable Diseases Manual. $16^{\text {th }}$ ed. Washington, DC: American Public Health Association; 1995.

3. Ponce-de-Leon S. The needs of developing countries and the resources required. J Hosp Infect 1991;18:376-81.

4. Ducel G, Fabry J, Nicolle L. Prevention of Hospital-Acquired Infections: A Practical Guide, WHO/CDS/CSR/EPH/2002.12. $2^{\text {nd }}$ ed. Geneva, Switzerland: World Health Organization; 2002.

5. Health Care - Associated Infections. World Health Organization. Available from: http://www.who.int/gpsc/country_work/gpsc_ccisc_ fact_sheet_en.pdf.

6. Bergogne-Berezinand E, Towner KJ. Acinetobacter spp. As nosocomial pathogens: Microbiological, clinical and epidemiological features. Clin Microbil Rev 1996;9(2):148-65.

7. Beck-Sagué CM, Jarvis WR, Brook JH, Culver DH, Potts A, Gay E, et al. Epidemic bacteremia due to Acinetobacter baumannii in five intensive care units. Am J Epidemiol 1990;132(4):723-33.

8. Villegas MV, Hartstein AI. Acinetobacter outbreaks, 1977-2000. Infect Control Hosp Epidemiol 2003;24(4):284-95.

9. Lortholary O, Fagon JY, Hoi AB, Slama MA, Pierre J, Giral P, et al. Nosocomial acquisition of multiresistant Acinetobacter baumannii: Risk factors and prognosis. Clin Infect Dis 1995;20(4):790-6.

10. Centers for Disease Control and Prevention (CDC). Overview of DrugResistant Acinetobacter Infections in Healthcare Settings. Available from: http://www.cdc.gov/ncidod/dhqp/ar_acinetobacter.html.

11. Tripathi PC, Gajbhiye SR, Agrawal GN. Clinical and antimicrobial profile of Acinetobacter spp.: An emerging nosocomial superbug. Adv Biomed Res 2014;3:13.

12. Agodi A, Zarrilli R, Barchitta M, Anzaldi A, Di Popolo A, Mattaliano A, et al. Alert surveillance of intensive care unit-acquired Acinetobacter infections in a Sicilian hospital. Clin Microbiol Infect 2006;12(3):241-7.

13. Shete VB, Ghadage DP, Muley VA, Bhore AV. Acinetobacter septicemia in neonates admitted to intensive care units. J Lab Physicians 2009;1(2):73-6. 